\title{
Study on the effective segmentation algorithm of image lesion areas in Department of Internal Medicine
}

\author{
Zhang Aiqin \\ Zaozhuang Vocational College of Science and Technology, shandong, tengzhou, 277500
}

Keywords: visceral adhesions; image segmentation; Department of internal medicine

\begin{abstract}
The effective segmentation algorithm of image lesion areas in Department of Internal Medicine is an important problem in the field of medical imaging. The current segmentation techniques of image lesion areas in Department of Internal Medicine are using the difference characters of edge pixels, due to visceral adhesions, the segmentation threshold is difficult to be accurately defined, and the precision is low. Therefore, this paper proposed a segmentation method of image lesion areas in Department of Internal Medicine based on improved cuckoo search algorithm to optimize maximum entropy. Firstly, by the maximum entropy method to find segmentation objective function of image lesion areas in Department of internal medicine, then the improved cuckoo search algorithm is adopted to optimize the objective function, find the best segmentation position of image lesion area in Department of internal medicine, so as to achieve image lesion area segmentation, finally multiple images of internal medicine are utilized to conduct simulation experiment, in order to test the performance of the proposed algorithm. The results show that, this method not only solves the defects existing in the traditional segmentation algorithm, while improving the precision of image lesion area of internal medicine, has better robustness, and better practical value.
\end{abstract}

\section{Introduction}

Image recognition technology of internal medicine has an important significance in improving disease diagnosis and the success rate of operation [1], which involves technologies of many disciplines like computer technology, image processing technology and imaging, and has broad prospects for developing [2]. Internal medicine image recognition includes 4 process, image acquisition, image segmentation, feature extraction and image recognition [3]. Among them, the segmentation method of image lesion area of internal medicine is the core part, segmentation accuracy of lesion area and normal area are the main factors that influence the identification accuracy. However, due to the constraint of image complexity of internal medicine [4], the current image lesion region segmentation method are still difficult to apply to the current situation, its complexity constraints are mainly including following two points: (1) for normal area and lesion area, large differences exists in the shape and color, there are still overlapping and adhesion phenomenon; (2) the image acquisition equipment affected by external conditions interference factors like light leading to complexity of the acquired image of internal medicine [5-7]. Therefore, image lesion region segmentation method of internal medicine is always a difficult problem in the field of medicine [8-10].

Aiming at the shortcoming of the traditional method, an image lesion region segmentation method of internal medicine based on improved cuckoo search algorithm for optimizing maximum entropy is proposed. Firstly, by the maximum entropy method to find segmentation objective function of image lesion areas in Department of internal medicine, then the improved cuckoo search algorithm is adopted to optimize the objective function, find the best segmentation position of image lesion area in Department of internal medicine, so as to achieve image lesion area segmentation, finally multiple images of internal medicine are utilized to conduct simulation experiment, in order to test the performance of the proposed algorithm. The results show that, this method not only solves the defects existing in the traditional segmentation algorithm, while improving the precision of image lesion area of internal medicine, has better robustness, and better practical value. 


\section{Image lesion region segmentation principle of internal medicine}

In clinical medical field, the image lesion region segmentation of internal medicine problem is a hot topic in the medical field, mainly depends on the different pixel feature at the connection of the lesion region and normal region to segment. Its principle is as follows:

The Gauss model in mixed Gauss models can be utilized to describe the feature of each pixel in the image of internal medicine, all pixels in the image of Department of internal medicine can be viewed as obtained through calculating the probability density function of internal medicine pixels in mixed pixel model. The probability density function of the pixel $x$ in the image of internal medicine, can be described with the following formula:

$$
p(x \mid \mu, \Sigma)=\sum_{k=1}^{K} \pi_{k} N\left(x_{i} \mid \mu_{k}, \Sigma_{k}\right)
$$

Among them, $\pi_{k}$ is the weight of mixed Gauss model, $\mu$ is the mean vector, $\Sigma$ is the covariance matrix of model. In the model, as long as these parameters are determined, the input image type of internal medicine will be determined. Typically, model parameters were determined by using EM. The method is a method of maximum likelihood, it through iterative processing method to solve the image pixel distribution parameters, concrete steps are as follows:

(1) Initialize parameters in Gauss model of image of internal medicine such as $\mu, \pi_{k}$ and $\Sigma$;

(2) With the parameter processed with initialization, maximum likelihood estimation is applied to hidden parameters in the model, the formula is as follows:

$$
\begin{gathered}
\gamma\left(\mathrm{z}_{n k}\right)=\frac{\pi_{k} N\left(x_{i} \mid \mu_{k}, \sum_{k}\right)}{\sum_{j=1}^{K} \pi_{j} N\left(x_{i} \mid \mu_{j}, \sum_{j}\right)} \\
N_{k}=\sum_{n=1}^{N} \gamma\left(\mathrm{z}_{n k}\right)
\end{gathered}
$$

(3) According to the estimation results in the step (2) to resolve the parameters in image pixels model of Department of internal medicine, the formula is as follows:

$$
\begin{gathered}
\mu_{k}=\frac{1}{N_{k}} \sum_{i=1}^{N} \gamma(i, k) x_{i} \\
\sum_{k}=\frac{1}{N_{k}} \sum_{i=1}^{N} \gamma(i, k)\left(x_{i}-\mu_{k}\right)\left(x-\mu_{k}\right)^{T} \\
\pi_{k}=\frac{N_{k}}{N}
\end{gathered}
$$

(4) The results obtained from resolved parameters will be input into the image pixel probability density function of Department of internal medicine to calculate, the formula is as follows:

$$
\ln p(x \mid \mu, \Sigma, \pi)=\sum_{n=1}^{N} \ln \left\{\sum_{k=1}^{K} \pi_{k} N\left(x_{i} \mid \mu_{k}, \sum_{k}\right)\right\}
$$

When the results of calculation satisfy convergence condition, then stop operation, so as to get the accurate image lesions region pixels calculation results of internal medicine, otherwise go back to step (2) to continue operation.

The specific steps of segmenting lesion region image of Department of internal medicine are as follows:

(1) After acquiring image of internal medicine, the filtering processing is done, RGB color is converted to CIELUV color, and the $L$ component graph are normalized, the formula is as follows:

$$
L(i, j)=255 \times\left(L(i, j)-L_{\min }\right) /\left(L_{\max }-L_{\min }\right)
$$

(2) For the $L$ component, the parameters of the model when $K=3$ is calculated, in order to avoid the defects of slow convergence rate and small color variation range within class, for the RGB color space of image of internal medicine to calculate parameters when $K=4$, the accurate image lesion region of internal medicine is obtained.

According to the method described above, the pixel probability density function of the image lesion region of internal medicine established with Gauss model, using the EM maximum likelihood method to re-estimate the parameters in the model, and the estimated results is input into the model to calculate, so as to acquire accurate the image lesion region segmentation results of internal medicine. However, the current image lesion region segmentation of internal medicine lesion is using the difference characters of edge pixels to segment, due to visceral adhesions, the 
segmentation threshold is difficult to be accurately defined, and the image lesion region segmentation accuracy of the Department of internal medicine is decreased.

\section{The segmentation method principle of the image lesion region of internal medicine based on maximum entropy of improved cuckoo search algorithm}

Description of improved cuckoo search algorithm. The bird's nest location is setting as $\mathrm{N}$ to represent the solution of the problem that the image lesion region segmentation of Department of internal medicine, each iteration of path and the position of the cuckoo searching for the bird's nest is updated according to the following formula:

$$
x_{i}^{t+1}=x_{i}^{t}+\alpha \oplus \mathrm{Levy}()
$$

In the formula, $x_{i}^{t+1}$ and $x_{i}^{t}$ respectively represents the position vector at $t$ generation and $t+1$ generation of the $i$-th bird's nest; $\alpha$ is step size adjustment factor; $\oplus$ stands for point to point multiplication, Lévy $(\lambda)$ is a random search path.

In order to realize the image lesion region segmentation of department of internal medicine, obtained simulation of flight jump path of $\operatorname{Lévy}(\lambda)$ is as follows:

$$
s=\frac{\mu}{|v|^{1 / \beta}}
$$

In the formula, $s$ is the Lévy flight path $\operatorname{Lévy}(\lambda), \beta=3 / 2, \mu, v$ are the random number of normal distribution.

The cuckoo search algorithm is a heuristic algorithm, it also has the defects like slow convergence speed at late stage, low convergence precision, easily into local minimum, therefore to improve CS algorithm, namely introducing differential evolution strategy in the CS algorithm, an improved CS algorithm (MCS) is proposed. The basic idea of the MCS algorithm: based on the cuckoo algorithm, the bird's nest location $x_{i}^{t}$ after evolution is not directly go into the next iteration $(t+1)$, but continue differential evolution, through the mechanism of variation to obtain the difference between individual and the excellent individuals in current population, so as to guide the evolutionary direction, and motivate to close to the best individual in the population, after new nest location is acquired, then move on to the next iteration, continuing to search and update location using the cuckoo basic algorithm, which increases the diversity of population, and avoid falling into local optimum and get the global optimal solution, but also improve the convergence speed and precision.

The maximum entropy method step of improved cuckoo search algorithm.

(1) Initialize the corresponding parameter of cuckoo algorithm.

(2) Calculate the number of pixels and the probability of gray value corresponding to image gray value of Department of internal medicine, and the gray value expectation of Department of internal medicine, and determine the range of the optimal threshold.

(3) Randomly initialize each nest location, and evaluate the adapt value of each nest's location.

(4) $\mathrm{Pa}$ and $\mathrm{R}$ were compared, according to the comparison result to change the location of the nest, to obtain a new set of bird's nest location.

(5) calculate the fitness value corresponding to new nest location, and compared with the fitness value corresponding to the last generation bird's nest position, if better, with the current fitness value to replace the fitness value of the last generation, and updates the corresponding nest location, then determine the current optimal location of the bird's nest and the optimal value.

(6) Mutation, crossover, evolutionary selection operation are processed to the new nest location as the initial points of difference evolutionary, to update the bird nest location, so as to obtain a new set of birds nest location;

(7) Evaluate fitness value corresponding to new group of bird's nest location in steps (6), by contrast, the current nest location and optimal value is determined;

(8) According to the termination condition, when the maximum number of iterations is achieved, the iteration is stopped, if not, then returns to step (4) to re-iterate for optimization.

(9) According to the optimal solution to obtain global optimal image lesions region segmentation 
threshold in Department of internal medicine.

\section{Experimental results and analysis}

In order to verify the validity of this algorithm, there is the need for an experiment. With the traditional algorithm and the proposed algorithm to segment the image lesion region of internal medicine, the effect of the image lesion region segmentation of Department of internal medicine using different algorithms can be shown in the following table:

Table 1 comparison of the effects obtained with different segmentation algorithm

\begin{tabular}{llll}
\hline algorithm & NESE & PSNR & MAE \\
\hline $\begin{array}{l}\text { EM model } \\
\begin{array}{l}\text { Improved } \\
\text { algorithm }\end{array}\end{array} \quad 0.25$ & 44.34 & 0.089 \\
$\begin{array}{l}\text { Fuzzy C means algorithm } \\
\text { The proposed algorithm }\end{array}$ & 0.18 & 46.78 & 0.064 \\
\hline
\end{tabular}

Table 2 Comparison of segmentation error with different algorithms

\begin{tabular}{llll}
\hline algorithm & Group 1 & Group 2 & Group 3 \\
\hline EM model & $1.57 \%$ & $1.59 \%$ & $1.48 \%$ \\
Meanshift algorithm & $0.65 \%$ & $0.63 \%$ & $0.68 \%$ \\
$\begin{array}{l}\text { Fuzzy C means } \\
\text { algorithm }\end{array}$ & $2.37 \%$ & $2.39 \%$ & $2.48 \%$ \\
The proposed algorithm & $0.31 \%$ & $0.33 \%$ & $0.31 \%$ \\
\hline
\end{tabular}

According to the result of the experiment table, it can be known that this algorithm for the image lesion region segmentation of internal medicine have better effect than the traditional algorithms, fully demonstrate the advantages of the proposed algorithm.

\section{Conclusions}

Aiming at the defect of traditional algorithm during image lesion area segmentation process of internal medicine. Therefore, this paper proposed a segmentation method of image lesion areas in Department of Internal Medicine based on improved cuckoo search algorithm to optimize maximum entropy. Firstly, by the maximum entropy method to find segmentation objective function of image lesion areas in Department of internal medicine, then the improved cuckoo search algorithm is adopted to optimize the objective function, find the best segmentation position of image lesion area in Department of internal medicine, so as to achieve image lesion area segmentation, finally multiple images of internal medicine are utilized to conduct simulation experiment, in order to test the performance of the proposed algorithm. The results show that, this method not only solves the defects existing in the traditional segmentation algorithm, while improving the precision of image lesion area of internal medicine, has better robustness, and better practical value.

\section{References}

[1] Wang Chuanzhong, Xiong Houjin. Intelligent blood cell image segmentation based on reinforcement learning [J]. Computer and modernization, 2013.2:31-34.

[2] Lin Kaiyan, Wu Junhui, Xu Lihong. Review on the methods of color image segmentation [J]. Journal of image and graphics: series A, 2005.1:1-10.

[3] Xu Ning, He Wenjun, Wu Xinzhong, et al. Design and application of performance evaluation scheme for blood white cells image automatic recognition system [J] Journal of Clinical Laboratory Sciences. 2013, (1): 76-77. 
[4] Ma Yide, Dai Ruolan, Li Lian. An automatic image segmentation method based on pulse coupled neural network and image entropy 2002.1:46-51. [J]. Journal of communications,

[5] Wang Jing, Feng Peng, Wei Biao, Chen Chao. Multi scale hybrid hierarchy fusion algorithm for biological cell image [J]. Acta optica Sinica, 2012, 32 (F12): 250-256.

[6] Ye Qixiang, Go Wen, Wang Weiqiang, Huang Tiejun. A color image segmentation algorithm based on the fusion of color and spatial information [J]. Journal of software, 2004.4:522-530.

[7] Pang Chunying, Liu Jikui, Han Lixi. The white blood cell image classification based on improved combination of FCM and LFP [J]. Journal of Image and Graphics, 2013, 18 (5): 545551.

[8] Jing Xiaojun, Cai Anni, Sun Jingao. An image segmentation algorithm based on twodimensional maximum between-cluster variance [J]. Journal of communications, 2001.4:71-76.

[9] Zhao Xuesong, Chen Shuzhen. Image segmentation method for integrated global binaryzation and edge detection [J]. Journal of Computer-Aided Design \& Computer Graphics, 2001.2:118121.

[10] Wang Jing, Cao Yiping, Cheng Xusheng. A method for image segmentation of white blood cell based on $\mathrm{H}$ histogram transformation [J]. Optics and optoelectronic technology, 2013, 11 (2): 74-78.

[11] Yong-gang tian, Dong Yi. Wavelet multi-scale segmentation algorithm in the application of cell images [J]. Computer simulation. 2011.11:255-301

[12] Yu-lin xu, zhang lei, Chen meters, Song Xinkun. Based on SIFT features and graph cut algorithm of image segmentation method research [J]. Computer measurement and control, 2009.12:2491-2493. 